\title{
THE EFFECT OF PEERS' AUTONOMY-SUPPORTIVE BEHAVIOUR ON ADOLESCENTS' PSYCHOLOGICAL NEED SATISFACTION, INTRINSIC MOTIVATION AND OBJECTIVELY MEASURED PHYSICAL ACTIVITY
}

\author{
Henri Tilga, Hanna Kalajas-Tilga, Vello Hein, \\ Lennart Raudsepp, ANdre KoKa \\ Institute of Sport Sciences and Physiotherapy, University of Tartu, Estonia
}

\begin{abstract}
Based on self-determination theory, adolescents' leisure-time (LT) physical activity (PA) behaviour may depend on the extent by which they perceive an environment as autonomy-supportive. The present study aimed to investigate whether adolescents' perception of autonomy-supportive behaviour from their peers is related to adolescents' objectively measured LT moderate-to-vigorous physical activity (MVPA) through perceived psychological needs satisfaction and intrinsic motivation towards PA. School students $(\mathrm{n}=215)$ aged between 12 to 15 years old (age: $13.20 \pm 0.96$ yrs) completed self-reported measures of perceived peers' autonomy support, psychological needs satisfaction, and intrinsic motivation in the context of LT PA. In addition, study participants wore an accelerometer (ActiGraph GT3X) to monitor their MVPA. Results of the structural equation modelling revealed that perceived autonomy support from peers had a significant and positive effect on perceptions of all three psychological need satisfaction in LT, which, in turn, had a significant and positive effect on adolescents' intrinsic motivation. Adolescents' LT PA was significantly predicted by their intrinsic motivation towards PA. Only one pathway through autonomy need satisfaction was revealed by which adolescents' perception of their peers' autonomy-supportive behaviour is related to intrinsic motivation and MVPA. The findings of the current study highlight the role of peers' autonomy-supportive behaviour on adolescents objectively measured PA through motivational processes in the context of LT.
\end{abstract}

Keywords: self-determination theory, autonomy support, psychological needs, intrinsic motivation, moderate-to-vigorous physical activity 


\section{INTRODUCTION}

During the last decade, an important issue is adolescents' physical activity (PA) as a sharp decrease in this area is evident $[9,11]$. There are a number of studies demonstrating the facilitative role of social-contextual factors such as perceived supportive behaviour from socializing agents like teachers, parents, and peers on leisure-time (LT) PA motivation, intention, and actual behaviour among adolescents $[2,13,16,17,19,22]$. While recognizing the importance of these studies in understanding the role of perceived supportive behaviours from significant others on adolescents' PA behaviour, one of the major limitations in these studies were relying on the subjective measures of PA behaviour. Several researchers $[1,17,31]$ have emphasized that objective PA assessment should be adopted as it is a more accurate method compared to self-reported measures. To date, to the best of our knowledge, there are only a few studies that have adopted an objective measure of PA when investigating relationships between the perceived supportive behaviour from socializing agents (e.g., teachers, parents, and peers) and PA behaviour among adolescents $[33,37,38]$. The present study was carried out with the aim to contribute to the existent literature by investigating the role of perceived autonomy-supportive behaviour from peers on objectively measured LT PA among Estonian adolescents.

The present study, as well as most of the previous studies examining the role of perceived supportive behaviour on adolescents' PA behaviour, used self-determination theory (SDT) [29] to guide the research hypotheses. SDT is a widely used theory of human motivation to explain the effect of social-contextual factors on individuals' psychological experiences and behaviour across different contexts [7] including education [36]. One of the SDT sub-theories is the basic psychological needs theory (BPNT) [8]. Based on BPNT, individuals have psychological needs for autonomy, competence and relatedness that hold the central role for understanding the associations between the social context and a person's motivation [8]. According to BPNT, it is individual's inner need to satisfy psychological needs for autonomy (i.e., to feel self-determined in one's actions), competence (i.e., to experience opportunities in which to express their capabilities), and relatedness (i.e., to feel connectedness to others) $[6,7,30]$. If these three psychological needs are fulfilled, people are more likely to experience activities as autonomous and express optimal psychological well-being [25]. Grounded in BPNT, significant others' behaviour can be viewed in terms of the extent to which it is autonomy-supportive and, therefore, whether it facilitates satisfaction of subordinates' psychological needs. The concept of autonomy support can be described as authoritative agents adopting certain behaviours 
that facilitate autonomy in the groups for whom they are in charge. To be autonomy-supportive, one should adopt perspectives and feelings of others, provide choice, give rationales, and encourage self-endorsed actions [21, 24].

As was already noted, there are only a few studies that have investigated the role of perceived autonomy-supportive behaviours from socializing agents (e.g., teachers, parents, and/or peers) on PA motivation and actual behaviour while adopting objective measures (e.g., pedometers and accelerometers) of PA [33, 37, 38]. Results of the study by Standage et al. [33], for example, showed that perceived autonomy support from physical education (PE) teacher was significantly and positively related to the LT PA motivation via $P E$ motivation. The indirect effect of perceived autonomy support on adolescents' PA measured as step counts via pedometers through motivational processes (i.e., basic needs satisfaction and motivation) in PE, however, was not significant. Similar findings were evident in studies conducted by Vierling et al. [37] and Wang [38]. Specifically, Vierling et al. [37] found that both perceived autonomy support form teachers and parents were significantly and positively related to PA motivation mediated by psychological need satisfaction, but not to adolescents' PA measured as step counts via pedometers. Finally, results of the study by Wang [38] revealed that perceived autonomy support from PE teachers, parents, and peers were all indirectly related to motivation towards LT PA mediated by psychological needs satisfaction. Nevertheless, the perceived autonomy-supportive behaviours from any of the socializing agents did not have a significant indirect effect on adolescents' objectively measured PA through the motivational processes including perceived psychological needs satisfaction and motivation towards LT PA. It should be noted that the latter studies adopted a specific approach in measuring motivation which is called relative autonomy index or selfdetermination index [28], consisting of weighted scores of different types of motivation introduced by SDT (i.e., intrinsic motivation, identified regulation, introjected regulation, extrinsic regulation, and amotivation) [29]. This approach, however, has lately been criticised as it could mask unique contribution of each type of motivational regulation [4].

The aim of the present study was to investigate whether adolescents' perception of autonomy-supportive behaviour from their peers is related to adolescents' objectively measured LT moderate-to-vigorous physical activity (MVPA) through their perceived satisfaction of the needs for autonomy, competence, and relatedness and intrinsic motivation. We used intrinsic motivation towards PA alone in this study as it has been considered as a prototypical form of autonomous motivation and reflects engaging in activities because doing so leads individuals to experience ownership over their 
action [29]. Moreover, research has shown that being intrinsically motivated towards particular activity will likely lead to most adaptive cognitive and behavioural outcomes. For example, intrinsic motivation towards PE has found to be related with higher concentration, preference to attempt challenging tasks, positive affect, and greater effort in classes [26, 32, 34].

Our hypothesised model is introduced in Figure 1. First, we hypothesised that adolescents' perceptions of their peers' autonomy-supportive behaviour will be directly related to adolescents' perceived psychological need satisfaction for autonomy, competence, and relatedness (H1). Second, we predicted that adolescents' perceived psychological need satisfaction for autonomy, competence, and relatedness will be directly related to adolescents' intrinsic motivation towards PA (H2). Third, we hypothesised that adolescents' intrinsic motivation towards PA will be directly related to adolescents' objectively measured LT MVPA (H3). Finally, we hypothesised that adolescents' perceptions of autonomy-supportive behaviour from their peers is related to objectively measured LT MVPA through psychological needs satisfaction for autonomy, competence, and relatedness and intrinsic motivation (H4).

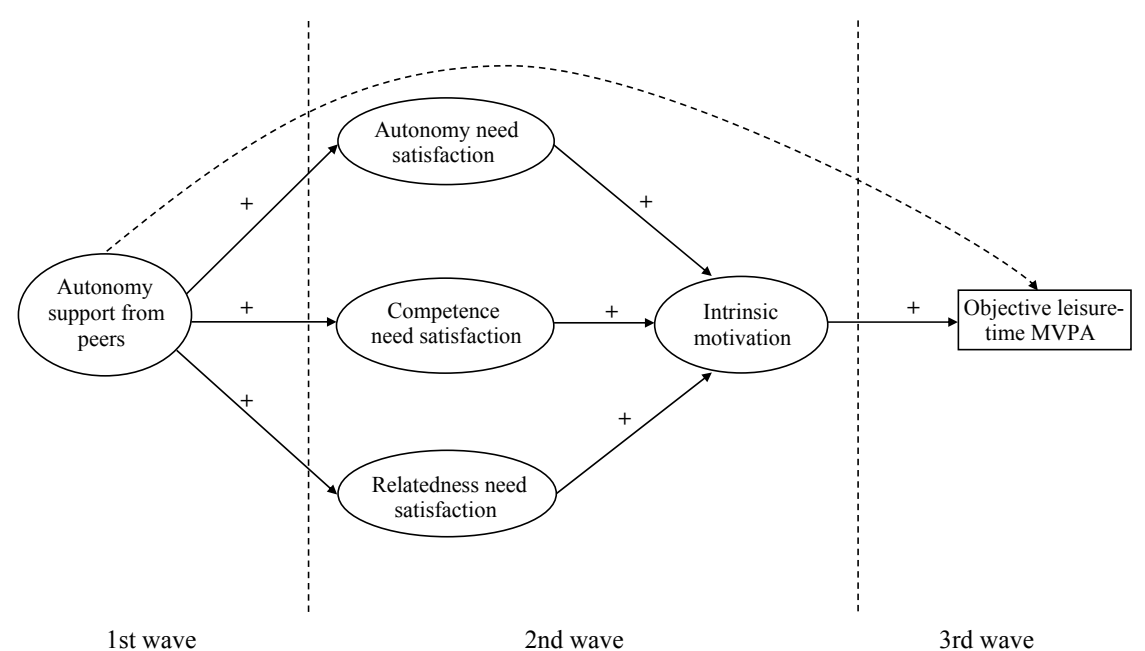

Figure 1. The proposed model demonstrating predicted relationships. Broken line indicate path set to be free in order to test indirect and mediation effects. 


\section{MATERIALS AND METHODS}

\section{Subjects and procedures}

The sample comprised of secondary school students ( $\mathrm{n}=215$; boys, $\mathrm{n}=61$, girls, $\mathrm{n}=154$ ) aged between 12 to 15 years old (age: $13.20 \pm 0.96 \mathrm{yrs}$ ). In the present study, a three-wave prospective correlational design was employed. In the first wave of data collection, a self-reported measure of adolescents' perceptions of autonomy-supportive behaviour from their peers in an LT context was administered. Five weeks later, a second-wave questionnaire was administered including measures of perceived need satisfaction and intrinsic motivation for LT PA. After five weeks, the third and final survey was conducted in which adolescents' objective PA was measured.

\section{Measures}

\section{Perceived peers' autonomy-supportive behaviour}

Adolescents' perception of their peers' autonomy-supportive behaviour was assessed using the short form of the Perceived Autonomy Support Scale for Exercise Settings (PASSES) [18] which contained four items. The tool is scored on a Likert scale of 1 (strongly disagree) to 7 (strongly agree). An example item is "I feel I am able to share my experience of active sports and/ or vigorous exercise with my friends". Previous studies have shown PASSES to be a valid and reliable measure [13].

\section{Perceived need satisfaction in leisure-time physical activity}

Adolescents' perceived need satisfaction during LT PA was assessed by three need satisfaction subscales (i.e., the need for autonomy, competence, and relatedness) from the Basic Psychological Need Scale and Need Frustration Scale (BPNSNF) [5] adapted to LT. Each subscale consisted of four items and was presented with a common stem, which reads "During my leisuretime..., followed by the set of items: need satisfaction for autonomy (e.g., "...I felt that the exercises reflect what I really want"), competence (e.g., "...I felt capable at what I did"), and relatedness (e.g., "...I felt that the friends I care about also cared about me"). The participants answered on a sevenpoint scale ranging from 1 (strongly disagree) to 7 (strongly agree). Previous studies have shown that BPNSNF is a valid and reliable measure $[14,35]$. 


\section{Intrinsic motivation towards leisure-time physical activity.}

Adolescents' intrinsic motivation towards LT PA was measured using a subscale for intrinsic motivation from an adapted version of the Perceived Locus of Causality Questionnaire (PLOCQ) [28]. The subscale consisted of two items (e.g., "I do physical activity during my free time because I enjoy doing physical activity"). The participants answered on a seven-point scale ranging from 1 (strongly disagree) to 7 (strongly agree). Previous studies have shown that PLOCQ is a valid and reliable measure [28].

\section{Physical activity.}

Adolescents' MVPA was measured using the Actigraph GT3X (ActiGraph LLC, Pensacola, FL). Students were asked to wear the accelerometer on their waist at all times for seven consecutive days, except when engaging in aquatic activities and while sleeping. Actigraph monitors were analysed using ActiLife software 6.13.3. The sampling interval was set at $15 \mathrm{~s}$. Accelerometer data were considered valid if over $600 \mathrm{~min}$ (10 hrs) of recorded data per day at least four days out of seven were present. Zero counts of consecutive 60 min were classified as non-wear time. The PA intensity level in accelerometers was measured using Evenson et al. [10] cutoff points.

\section{Statistical analysis}

The statistical packages SPSS Version 23.0 and AMOS Version 23.0 were used for complete data analyses. Goodness-of-fit indices suggested by $\mathrm{Hu}$ and Bentler [20] were used to assess how well did data fit the proposed scales. We used the following indices the comparative fit index (CFI), the Bentler-Bonett non-normed fit index (NNFI), and the root mean square error of approximation (RMSEA). An acceptable fit of the data is considered by values of $>0.90$ for the CFI and NNFI, and value $<0.08$ for the RMSEA [20]. The adequacy of a multi-factor CFA that included five latent constructs and 18 indicators was estimated. Next, the structural equation modelling was used to test the study hypotheses. In the structural equation model, all latent variables were indicated by multiple items.

\section{RESULTS}

\section{Preliminary analysis}

A total of 424 adolescents (158 male, 266 female) completed the first wave of data collection. During the second and third wave of data collection, there was a drop out of 18 and 34 adolescents, respectively, that was primarily due 
to being absent from school. An additional 157 adolescents were excluded from the study because their accelerometer data did not cover wearing the device at least 10 hours a day during four consecutive days. Finally, there were 215 participants who provided complete data suitable for statistical analysis. Values for skewness ranged from -1.42 to 0.58 and values for kurtosis ranged from -0.26 to 1.85 , suggesting that all items were within acceptable ranges [12]. Mardia's normalized coefficient revealed that the data deviated from multivariate normality, and thus, bootstrapping procedure was used to generate standard errors of optimal precision with 5000 resamples $[3,27]$.

\section{The measurement model, descriptive statistics and reliability}

Results of the measurement model (i.e., a model that includes all the scales) indicated acceptable goodness-of-fit statistics: $\chi^{2}=270.52, \mathrm{df}=121, \mathrm{p}<0.001$; $\mathrm{CFI}=0.94 ; \mathrm{NNFI}=0.93 ; \mathrm{RMSEA}=0.08$. Table 1 presents correlations for all study variables, descriptive statistics and Cronbach's a coefficients. The constructs of perceived autonomy support from peers, adolescents' need satisfaction for autonomy, competence, and relatedness and intrinsic motivation were all positively correlated with each other. Only students' intrinsic motivation towards PA was significantly and positively correlated with adolescents' LT MVPA.

Table 1. Descriptive statistics, scale reliabilities and correlations among latent study variables.

\begin{tabular}{lcccccc}
\hline \multirow{2}{*}{ Variable } & \multicolumn{7}{c}{ Correlation } \\
\cline { 2 - 7 } & 1 & 2 & 3 & 4 & 5 & 6 \\
\hline 1. Autonomy support from peers & \multicolumn{7}{c}{0} & & & & \\
\hline 2. Autonomy need satisfaction & $0.44^{* *}$ & & & & \\
\hline 3. Competence need satisfaction & $0.40^{* *}$ & $0.87^{* *}$ \\
\hline 4. Relatedness need satisfaction & $0.51^{* *}$ & $0.70^{* *}$ & $0.72^{* *}$ \\
\hline 5. Intrinsic motivation & $0.44^{* *}$ & $0.76^{* *}$ & $0.76^{* *}$ & $0.52^{* *}$ & & \\
\hline 6. MVPA & 0.13 & 0.06 & 0.10 & 0.01 & $0.19^{*}$ \\
\hline M & 5.23 & 5.69 & 5.61 & 5.39 & 5.48 & 39.77 \\
\hline SD & 1.31 & 1.08 & 1.07 & 1.26 & 1.39 & 19.24 \\
\hline a & 0.87 & 0.89 & 0.86 & 0.90 & 0.87 & N/A \\
\hline
\end{tabular}

MVPA - moderate-to-vigorous physical activity; M - mean; SD - standard deviation; a Cronbach's a coefficients

${ }^{*} \mathrm{p}<0.01 ;{ }^{* *} \mathrm{p}<0.001$ 


\section{Main analysis}

Results of the structural model indicated acceptable goodness-of-fit statistics: $\chi^{2}=280.54, \mathrm{df}=125, \mathrm{p}<0.001 ; \mathrm{CFI}=0.94 ; \mathrm{NNFI}=0.93$; RMSEA $=0.08$. In the hypothesised model (see Figure 2), perceived autonomy support from peers had direct and positive effects on the students' need satisfaction for autonomy $(\beta=0.45, \mathrm{p}<0.001)$, competence $(\beta=0.42, \mathrm{p}<0.001)$, and relatedness $(\beta=0.51, p<0.001)$. The direct relationship between perceived autonomy support from peers and adolescents' MVPA was not significant. Next, significant relationships were found between adolescents' intrinsic motivation and need satisfaction for autonomy $(\beta=0.47, p<0.001)$, competence $(\beta=0.43$, $\mathrm{p}<0.001)$, and relatedness $(\beta=0.63, \mathrm{p}<0.001)$. Finally, adolescents' intrinsic motivation had direct and positive effect on the MVPA $(\beta=0.15, p=0.05)$. An analysis pertaining to specific indirect effects revealed that perceived autonomy support from peers was related to adolescents' MVPA mediated by autonomy need satisfaction and intrinsic motivation $(\beta=0.56$, $\mathrm{p}=0.05$ ), but not by competence need satisfaction and intrinsic motivation $(\beta=0.46, p=0.09)$ or by relatedness need satisfaction and intrinsic motivation $(\beta=-0.15, p=0.14)$.

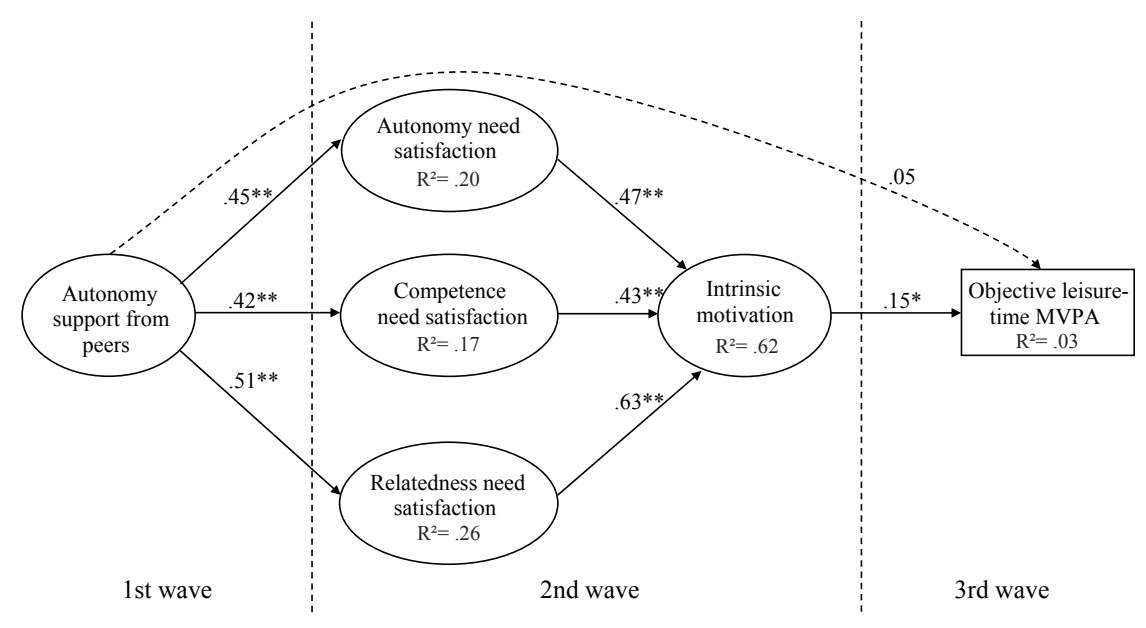

Figure 2. The structural equation model measuring the relationships between students' perception of autonomy-supportive behaviour and leisure-time physical activity through leisure-time need satisfaction for autonomy, competence and relatedness, and intrinsic motivation. ${ }^{* *} p<0.001,{ }^{*} p<0.05$. 


\section{DISCUSSION}

The current study was conducted to explain the effect of adolescents' perception of autonomy-supportive behaviour from their peers on adolescents' objectively measured MVPA through perceived psychological needs satisfaction and intrinsic motivation towards PA. Specifically, only one pathway through autonomy need satisfaction was identified by which adolescents' perceived autonomy-supportive behaviour from their peers is related to adolescents' objectively measured MVPA. The findings of the current study highlight the important role of peers' autonomy-supportive behaviour as it significantly contributed to adolescents' objective MVPA through perceived satisfaction of the need for autonomy and intrinsic motivation in the context of LT.

In line with our hypothesis $\mathrm{H} 1$, adolescents' perception of autonomysupportive behaviour from their peers was related to adolescents' perceived psychological needs for autonomy, competence, and relatedness in a context of LT PA. This finding is similar with previous studies indicating that autonomy support from peers fosters the three psychological needs for autonomy, competence, and relatedness [13, 22, 23, 38]. It is noteworthy that in the present study the magnitude of the effects between the aforementioned variables was different. Specifically, in line with previous studies by Koka [22, 23] and González-Cutre et al. [13], perceived autonomy-supportive behaviour from peers had the strongest effect on students' relatedness need satisfaction, followed by autonomy and competence need satisfaction. The possible explanation for the strongest relationship between perceived peer autonomy support and relatedness need satisfaction might be that close relationships with peers are particularly important at this age. Additionally, it might be that peers' autonomy supportive behaviour might satisfy more relatedness needs than other basic psychological needs, as the source is friends and the with whom the relationship is most likely closer than with other significant others such as teachers or parents.

In line with our hypothesis $\mathrm{H} 2$, adolescents' psychological needs for autonomy, competence, and relatedness were related to their intrinsic motivation towards LT PA. In some extent, this is similar with previous work [38] demonstrating that the fulfilment of psychological needs for autonomy, competence, and relatedness in a context of LT PA contribute to the facilitation of autonomous motivation towards PA measured as relative autonomy index. In the current study, it was found that the strongest predictor of adolescents' intrinsic motivation was relatedness need satisfaction, followed by autonomy and competence need satisfaction. As adolescents' 
intrinsic motivation was measured in the context of LT, it can be expected that relatedness need satisfaction might be the strongest direct predictor. This finding, however, is different from the results found by González-Cutre et al. [13] and Wang [38]. While significant, albeit small, the relationship between perceived relatedness need satisfaction and relative autonomy index was found in a study of Wang [38], no significant relationship was found between relatedness need satisfaction and relative autonomy index in a study of González-Cutre et al. [13]. Several possible explanations for this discrepancy can be discussed. First, as relative autonomy index consists of different types of motivational regulations, there might be other sources for adolescents' combined motivation which drive them to act than perceived relatedness need satisfaction. Another possible explanation might be that the reason for adolescents to communicate with other peers usually comes from a pure inherent interest, an intrinsically motivated act, as there is usually no direct obligation to interact with each other at this age.

In line with our hypothesis $\mathrm{H} 3$, adolescents' intrinsic motivation towards LT PA had a direct and significant effect on their objectively measured MVPA. The reason for this might be that during their free time adolescents usually can decide by their own free will whether to be physically active. In addition, one might argue that an activity has to be intrinsically motivating for adolescents to act on their free will. This is different from a previous research [22] in which no direct and significant relationship between autonomous motivation and LT PA was found. The autonomous motivation, as measured in a study by Koka [22], consisted of several motivational regulations which might have masked the unique contribution of each type of motivational regulations [4].

Finally, only one full pathway was identified by which adolescents' perceptions of their peers' autonomy-supportive behaviour were related to adolescents' intrinsic motivation towards PA and objectively measured MVPA. In line with our main hypothesis $\mathrm{H} 4$, adolescents' need satisfaction for autonomy was found to be the mediator between those variables. This finding is different from previous studies $[33,38]$ in which no significant indirect effect on adolescents' objectively measured PA through the motivational processes including perceived psychological needs satisfaction and motivation towards LT PA was found. The reason for this might be that in the aforementioned studies motivation was measured by relative autonomy index or self-determination index that might mask the unique contribution of each type of motivational regulations [4]. The possible explanation for the significant pathway from perceived autonomy-supportive behaviour from peers to adolescents' objectively measured PA found in the present study might be 
that that intrinsic motivation as a mediator was measured which enabled to estimate the role of motivation in a more specific way. However, adolescents' need satisfaction for competence and relatedness were not found to act as mediators between the pathway by which adolescents' perceptions of their peers' autonomy-supportive behaviour was related to adolescents' intrinsic motivation towards PA and objectively measured MVPA. It is noteworthy that despite the strongest direct relationships were found between adolescents' perception of autonomy-supportive behaviour from peers and relatedness need satisfaction and, in turn, between relatedness need satisfaction and intrinsic motivation, there was no significant indirect pathway from adolescents' perception of autonomy-supportive behaviour from peers to adolescents' intrinsic motivation and objectively measured MVPA. The possible explanation of why only the pathway through autonomy need satisfaction was significant might be that adolescents' perception of peers' autonomy-supportive behaviour was measured.

A few limitations of the current study should be noted to contextualise our findings and stipulate future research. Firstly, there is a big difference between the number of boys and girls participating in the current study. It might be due to the fact that girls are more willing to participate in such kind of surveys. Secondly, ActiGraph accelerometers lack the information about certain activities such as swimming or weightlifting. Future studies measuring students' objective PA could use accelerometer diaries to provide more detailed information. Thirdly, the current study did not estimate an opposite aspect of autonomy supportive behaviours from peers (e.g., controlling behaviour) and its relationship with objective LT PA through potential mediators such as perceived psychological need frustration and extrinsic motivation. Specifically, previous studies have shown that even when significant others are autonomy-supportive, and they also use controlling techniques concurrently, it will lead to maladaptive outcomes [15]. Therefore, it is important to measure more aspects of peers' behaviour, psychological needs and different types of motivation to provide more accurate knowledge of the determinants of adolescents' objectively measured MVPA.

To sum up, the current study adds to the existing literature by specifying the mediating role of different psychological need satisfaction on the relationship from adolescents' autonomy-supportive behaviour to their intrinsic motivation and objectively measured MVPA. As expected, direct and significant positive pathways were found in the proposed model between each construct in the theoretically proposed sequence. However, only one full pathway through autonomy need satisfaction was found from adolescents' perception of autonomy-supportive behaviour from peers to 
adolescents' intrinsic motivation and objectively measured MVPA. The findings of the current study highlight the role of peers' autonomy-supportive behaviour as it significantly contributes to adolescents' objective MVPA through perceived satisfaction of the need for autonomy and intrinsic motivation in the context of LT.

\section{ACKNOWLEDGEMENT}

This work was supported by the Estonian Research Council grant (PUT 1542).

\section{REFERENCES}

1. Adams SA, Matthews CE, Ebbeling CB, Moore CG, Cunningham JE, Fulton J, Hebert JR. (2005) The effect of social desirability and social approval on selfreports of physical activity. Am J Epidemiol, 161: 389-398.

2. Barkoukis V, Hagger MS, Lambropoulos G, Torbatzoudis H. (2010) Extending the trans-contextual model in physical education and leisure-time contexts: Examining the role of basic psychological need satisfaction. Brit J Educ Psychol, 80: 647-670.

3. Byrne BM. (2010) Structural equation modeling with AMOS: Basic concepts, applications, and programming (2nd ed). New York, NY: Routledge.

4. Chemolli E, Gagne M. (2014) Evidence against the continuum structure underlying motivation measures derived from self-determination theory. Psychol Assessment, 26: 575-585.

5. Chen B, Vansteenkiste M, Beyers W, Boone L, Deci EL, Van der Kaap-Deeder J, Duriez B, Lens W, Matos L, Mouratidis A, Ryan RM, Sheldon KM, Soenens B, Van Petegem S, Verstuyf J. (2015) Basic psychological need satisfaction, need frustration, and need strength across four cultures. Motiv Emotion, 39: 216-236.

6. Deci EL, Ryan RM. (1985) Intrinsic motivation and self-determination in human behavior. New York: Plenum.

7. Deci EL, Ryan RM. (2000) The "what" and "why" of goal pursuits: Human needs and the self-determination of behavior. Psychol Inq, 11: 227-268.

8. Deci EL, Ryan RM. (2002) Self-determination research: reflections and future directions. In Deci EL, Ryan RM. (eds), Handbook of self-determination research. Rochester, NY: University of Rochester Press, 431-441.

9. Dishman RK, McIver KL, Dowda M, Pate RR. (2018) Declining physical activity and motivation from middle school to high school. Med Sci Sport Exer, 50: 1206-1215. 
10. Evenson KR, Catellier DJ, Gill K, Ondrak KS, McMurray RG. (2008) Calibration of two objective measures of physical activity for children. J Sport Sci, 26: 1557-1565.

11. Fernandes HM. (2018) Physical activity levels in Portuguese adolescents: A 10-year trend analysis (2006-2016). J Sci Med Sport, 21: 185-189.

12. George D, Mallery M. (2010) SPSS for windows step by step: A simple guide and reference. Boston, MA: Pearson.

13. González-Cutre D, Sicilia A, Beas-Jimenez M, Hagger MS. (2014) Broadening the trans-contextual model of motivation: A study with Spanish adolescents. Scand J Med Sci Spor, 24: 306-319.

14. Haerens L, Aelterman N, Vansteenkiste M, Soenens B, Van Petegem S. (2015) Do perceived autonomy-supportive and controlling teaching relate to physical education students' motivational experiences through unique pathways? Distinguishing between the bright and dark side of motivation. Psychol Sport Exerc, 16: 26-36.

15. Haerens L, Vansteenkiste M, De Meester A, Delrue J, Tallir I, Vande G, Goris BW, Aelterman N. (2017) Different combinations of perceived autonomy support and control: identifying the most optimal motivating style. Phys Educ Sport Peda, 23: 16-36.

16. Hagger MS, Chatzisarantis NLD, Barkoukis V, Wang CKJ, Baranowski J. (2005) Perceived autonomy support in physical education and leisure-time physical activity: A cross-cultural evaluation of the trans-contextual model. J Educ Psychol, 97: 376-390.

17. Hagger MS, Chatzisarantis NLD, Culverhouse T, Biddle SJH. (2003) The processes by which perceived autonomy support in physical education promotes leisure-time physical activity intentions and behavior: A trans-contextual model. J Educ Psychol, 95: 784-795.

18. Hagger MS, Chatzisarantis, NLD, Hein V, Pihu M, Soos I, Karsai I. (2007) The perceived autonomy support scale for exercise settings (PASSES): Development, validity, and cross-cultural invariance in young people. Psychol Sport Exerc, 8: 632-653.

19. Hagger MS, Chatzisarantis NLD, Hein V, Soos I, Lintunen T, Karsai I, Leemans S. (2009) Teacher, peer and parent autonomy support in physical education and leisure-time physical activity: A trans-contextual model of motivation in four nations. Psychol Health, 24: 689-711.

20. Hu LT, Bentler PM. (1999) Cutoff criteria for fit indexes in covariance structure analysis: Conventional criteria versus new alternatives. Struct Equ Modeling, 6: $1-55$.

21. Jang H, Reeve J, Deci EL. (2010) Engaging students in learning activities: It is not autonomy support or structure but autonomy support and structure. J Educ Psychol, 102: 588-600. 
22. Koka A. (2013) The effect of teacher and peers need support on students' motivation in physical education and its relationship to leisure time physical activity. Acta Kinesiol Univ Tartu, 19: 48-62.

23. Koka A. (2014) The relative roles of teachers and peers on students' motivation in physical education and its relationship to self-esteem and health-related quality of life. Int J Sport Psychol, 45: 187-213.

24. McLachlan S, Hagger MS. (2010) Effects of an autonomy-supportive intervention on tutor behaviors in a higher education context. Teach Teach Educ, 26: 1205-1211.

25. Mouratidis AA, Vansteenkiste M, Sideridis G, Lens W. (2011) Vitality and interest-enjoyment as a function of class-to-class variation in need-supportive teaching and pupils' autonomous motivation. J Educ Psychol, 103: 353-366.

26. Ntoumanis N. (2001) A self-determination approach to the understanding of motivation in physical education. Brit J Educ Psychol, 71: 225-242.

27. Preacher KJ, Hayes AF. (2008) Asymptotic and resampling strategies for assessing and comparing indirect effects in multiple mediator models. Behav Res Methods, 40: 879-891.

28. Ryan RM, Connell JP. (1989) Perceived locus of causality and internalization: Examining reasons for acting in two domains. J Pers Soc Psychol, 57: 749-761.

29. Ryan RM, Deci EL. (2017) Self-determination theory: Basic psychological needs in motivation, development, and wellness. New York: Guilford Press.

30. Sheldon KM, Elliot, AJ, Kim Y, Kasser T. (2001) What's satisfying about satisfying events? Comparing ten candidate psychological needs. J Pers Soc Psychol, 80: 325-339.

31. Standage M, Duda JL, Ntoumanis N. (2003) A model of contextual motivation in physical education: Using constructs from self-determination and achievement goal theories to predict physical activity intentions. J Educ Psychol, 95: 97-110.

32. Standage M, Duda JL, Ntoumanis N. (2005) A test of self-determination theory in school physical education. Brit J Educ Psychol, 75: 411-433.

33. Standage M, Gillison FB, Ntoumanis N, Treasure DC. (2012) Predicting students' physical activity and health-related well-being: A prospective cross-domain investigation of motivation across school physical education and exercise settings. J Sport Exercise Psy, 34: 37-60.

34. Taylor IM, Ntoumanis N, Standage M, Spray CM. (2010) Motivational predictors of physical education students' effort, exercise intentions, and leisure-time physical activity: A multilevel linear growth analysis. J Sport Exercise Psy, 32: 99-120.

35. Tilga H, Hein V, Koka A. (2017) Measuring the perception of the teachers' autonomy-supportive behavior in physical education: Development and initial validation of a multi-dimensional instrument. Meas Phys Educ Exerc Sci, 4: 244-255.

36. Van de Berghe L, Vansteenkiste M, Cardon G, Kirk D, Haerens L. (2014) Research on self-determination in physical education: Key findings and proposals for future research. Phys Educ Sport Peda, 1: 97-121. 
37. Vierling K, Standage M, Treasure DC. (2007) Predicting attitudes and physical activity in an "at-risk" minority youth sample: A test of the self-determination theory. Psychol Sport Exerc, 8: 795-817.

38. Wang L. (2017) Using the self-determination theory to understand Chinese adolescent leisure-time physical activity. Eur J Sport Sci, 17: 453-461.

\section{Correspondence to:}

Henri Tilga

Institute of Sport Sciences and Physiotherapy, Faculty of Medicine

University of Tartu, 5 Jakobi St., Tartu, 51014

E-mail: henri.tilga@ut.ee 